Publ. RIMS, Kyolo Univ.

15 (1979), 631-651

\title{
Mixed Problems in a Quarter Space for the Wave Equation with a Singular Oblique Derivative
}

By

\author{
Hideo SOGA ${ }^{*}$
}

\section{品1. Hentroduction}

Let us consider the mixed problem

(1.1) $\left\{\begin{array}{c}\square u \equiv\left(\frac{\partial^{2}}{\partial t^{2}}-\frac{\partial^{2}}{\partial x^{2}}-\frac{\partial^{2}}{\partial y^{2}}\right) u(x, y, t)=f(x, y, t) \text { in } \mathbb{R}_{+}^{2} \times(0, T), \\ \left.B u \equiv\left(\frac{\partial}{\partial y}+\dot{\varphi}(y) \frac{\partial}{\partial x}\right) u\right|_{x=0}=g(y, t) \text { on } \mathbb{R}^{1} \times(0, T), \\ \left.\frac{\partial u}{\partial t}\right|_{t=0}=u_{1}(x, y) \text { on } \mathbb{R}_{+}^{2}, \\ \left.u\right|_{t=0}=u u_{0}(x, y) \text { on } \mathbb{R}_{+}^{2},\end{array}\right.$

where $\mathbb{R}_{+}^{2}=\left\{(x, y): x>0, y \in \mathbb{R}^{1}\right\}$ and $\psi(y)$ is a real-valued function belonging to $\mathscr{B}^{\infty}\left(\mathbb{R}^{1}\right)=\left\{\chi(y) \in C^{\infty}\left(\mathbb{P}^{1}\right) ; \sup _{y \in \mathbb{R}}\left|\frac{d^{j} \chi}{d y_{j}}(y)\right|<\infty, j=0,1,2, \cdots\right\}$. When the boundary operator is non-characteristic (i.e. $\inf _{y \in \mathbb{R}}|\psi(y)|>0$ ), it is known that (1.1) is well-posed (in the sense that the solution exists uniquely) and has a finite propagation speed (cf. Ikawa [2]).

In the present paper we shall study (1.1) in a singular case, that is, $\psi(y)$ may vanish in a finite interval. Our main result is the following

Theorem. Let $\psi(y)$ be of the form $\varphi(y)^{2}$ or $-\varphi(y)^{2}$ where $\varphi(y)$ $\left(\in \mathscr{B}^{\infty}\right)$ is real-valued and $\inf _{|y| \geq y_{0}}|\varphi(y)|>0$ for a large $y_{0}$. Then the problem (1.1) has a unique solution $u(x, y, t)$ in $C^{\infty}\left(\overline{\mathbb{R}_{+}^{2}} \times[0, T]\right)$ for any $\left(u u_{0}(x, y), u_{1}(x, y), f(x, y, t), g(y, t)\right) \in C^{\infty}\left(\overline{\mathbb{R}_{+}^{2}}\right) \times C^{\infty}\left(\overline{\mathbb{R}_{+}^{2}}\right) \times C^{\infty}\left(\overline{\mathbb{R}_{+}^{2}}\right.$ $\times[0, T]) \times C^{\infty}\left(\mathbb{R}^{1} \times[0, T]\right)$ saitsfying the compatibility condition of infinite order. Furthermore, the domain of dependence is finite.

Communicated by S. Matsuura, December 14, 1976.

* Department of Mathematics, Faculty of Education, Ibaraki University. 
Here 'the compatibility condition of order $m$ ' means that the data $\left(u_{0}, u_{1}, f, g\right)$ satisfy

$$
\left.B u_{j} \equiv\left(\frac{\partial}{\partial y}+\psi(y) \frac{\partial}{\partial x}\right) u_{j}\right|_{x=0}=\left.\frac{\partial^{j} g}{\partial t^{j}}\right|_{t=0} \quad \text { on } \quad \boldsymbol{R}_{y}^{1}
$$

for $\quad j=0,1,2, \cdots, m \quad$ where $\quad u_{j}=\left(\frac{\partial^{2}}{\partial x^{2}}+\frac{\partial^{2}}{\partial y^{2}}\right) u_{j-2}+\left.\frac{\partial^{j-2} f}{\partial t^{j-2}}\right|_{t=0}(j=2,3, \cdots$, $m)$.

Freezing the boundary operator of (1.1) at a point where $\psi(y)=0$, we have the problem

$$
\left\{\begin{array}{l}
\square u(x, y, t)=f(x, y, t) \quad \text { in } \quad \boldsymbol{R}_{+}^{2} \times(0, T), \\
\left.\frac{\partial u}{\partial y}\right|_{x=0}=g(y, t) \quad \text { on } \quad \boldsymbol{R}^{1} \times(0, T), \\
\left.\frac{\partial u}{\partial t}\right|_{t=0}=u_{1}(x, y) \text { on } \quad \boldsymbol{R}_{+}^{2}, \\
\left.u\right|_{t=0}=u_{0}(x, y) \text { on } \quad \boldsymbol{R}_{+}^{2} .
\end{array}\right.
$$

Obviously this problem is not well-posed (cf. Sakamoto [7]). Our result shows that in a certain case the existence of the solution holds even if the mixed problem for the frozen operator is not well-posed at some points.

Our method is as follows: Consider the Dirichlet problem

$$
\left\{\begin{array}{lll}
\square w(x, y, t)=0 & \text { in } & \boldsymbol{R}_{+}^{2} \times \boldsymbol{R}^{1}, \\
\left.w\right|_{x=0}=h(y, t) & \text { on } & \boldsymbol{R}^{1} \times \boldsymbol{R}^{1}
\end{array}\right.
$$

and set

$$
T h=B w .
$$

Then we can reduce the original problem (1.1) to the equation $T h=g$ on the boundary, and investigate it by means of the methods for pseudodifferential operators.

In $\S 3$ we study the solvability and the estimate of the equation $T h=g$, and show the unique existence of the solution of (1.1). Section 4 is devoted to a study of the domain of dependence. The problem (1.1) has not a finite propagation speed, but the domain of dependence is finite. Namely, for any $\left(x_{0}, y_{0}, t_{0}\right) \in \overline{\boldsymbol{R}_{+}^{2}} \times(0, T)$ there exists a bounded set $D$ of $\overline{\boldsymbol{R}_{+}^{2}} \times[0, T)$ such that if the data $\left(\iota_{0}, u_{1}, f, g\right)$ satisfy 


$$
\left\{\begin{array}{lll}
f(x, y, t)=0 & \text { on } & D \cap\left\{x>0,0<t<t_{0}\right\}, \\
g(y, t)=0 & \text { on } D \cap\left\{x=0,0<t<t_{0}\right\}, \\
u_{0}(x, y)=u_{1}(x, y)=0 & \text { on } D \cap\{x>0, t=0\}
\end{array}\right.
$$

the solution $u(x, y, t)$ is equal to zero on $D \cap\left\{x>0,0<t<t_{0}\right\}$. In $\S 5$ we show that the mixed problem (1.1) is not well-posed if $\psi(0)=0$, $\psi(y)>0$ for $y<0$ and $\psi(y)<0$ for $y>0$.

We note that our results are valid also in the case where the boundary is $\mathbb{R}_{y}^{n}(n \geqq 2)$ if the boundary operator is of the form $\frac{\partial}{\partial y_{1}}+a_{2} \frac{\partial}{\partial y_{2}}+\cdots$ $+a_{n} \frac{\partial}{\partial y_{n}}+\psi\left(y_{1}\right) \frac{\partial}{\partial x}$ ( $a_{i}$ is a real constant $)$.

The author wishes to express his sincere gratitude to Professor M. Ikawa for his much advice.

\section{§ 2. Prelimimaries}

At first we state notations and some properties of pseudo-differential operators with a complex parameter $\tau=\gamma+i \sigma\left(\gamma>0, \sigma \in \mathbb{R}^{1}\right)$. We denote by $S_{\rho, \delta}^{m}(m \in \mathbb{R}, 0 \leqq \delta \leqq \rho \leqq 1, \delta<1)$ the set of functions $p(y, \eta, \tau) \in C^{\infty}\left(\mathbb{R}^{2}(y, \eta)\right)$ with the parameter $\tau$ satisfying for all non-negative integers $\alpha, \beta$

$$
\frac{\partial^{\alpha+\beta}}{\partial y^{\beta} \partial \eta^{\alpha}} p(y, \eta, \tau) \mid \leqq C_{\alpha \beta}\left(\eta^{2}+|\tau|^{2}\right)^{(1 / 2)(m-\rho \alpha+\delta \beta)},
$$

where the constant $C_{\alpha \beta}$ does not depend on $\tau$ when $\gamma=\operatorname{Re} \tau \geqq 1$. For $p(y, \eta, \tau) \in S_{\rho, \delta}^{m}$ we define a pseudo-differential operator $p=p\left(y, D_{y}, \tau\right)$ by

$$
p u=p\left(y, D_{y}, \tau\right) u(y)=\int e^{i y \eta} p(y, \eta, \tau) \hat{u}(\eta) d \eta, \quad u(y) \in \mathscr{S},
$$

where $d \eta=\frac{1}{2 \pi} d \eta, \mathscr{S}$ is the space of rapidly decreasing functions and $\hat{u}(\eta)$ is the Fourier transform of $u(v)$, that is,

$$
\hat{u}(\eta)=\mathscr{I}[u]=\int e^{-i y \eta} u(v) d y .
$$

Define the norm $\|\cdot\|_{s}(s \in \mathbb{R})$ with the parameter $\tau$ by

$$
\|u\|_{s}^{2}=\int\left(\eta^{2}+|\tau|^{2}\right)^{s}|\hat{u}(\eta)|^{2} d \eta
$$

As is well known, the estimate 


$$
\|p u\|_{0} \leqq C\|u\|_{m}, \quad u \in \mathscr{S}
$$

holds for $p(y, \eta, \tau) \in S_{\rho, \delta}^{m}$, where the constant $C$ is independent of $\tau(\gamma$ $=\operatorname{Re} \tau \geqq 1$ ) (cf. Calderón-Vaillancourt [1] or Theorem 1.6 in Chapter 7 of [6]). Let $\chi(y, \eta) \in \mathscr{S}\left(\mathbb{R}^{2}\right)$ and $\chi(0,0)=1$, and define the oscillatory integral Os- $\iint e^{-i y \eta} p(y, \eta, \tau) d y d \eta$ for $p(y, \eta, \tau) \in S_{\rho, \delta}^{m}$ by

$$
\lim _{\varepsilon \rightarrow 0} \iint e^{-i y \eta} \chi(\varepsilon y, \varepsilon \eta) p(y, \eta, \tau) d y d \eta
$$

Then we have the formula (integration by parts)

$$
\begin{aligned}
& \text { Os- } \iint e^{-i y \eta} p(y, \eta, \tau) \eta^{\alpha} d y d \eta \\
& \quad=\text { Os- } \iint e^{-i y \eta} D_{y}^{\alpha} p(y, \eta, \tau) d y d \eta \quad\left(D_{y}^{\alpha}=\left(-i \frac{\partial}{\partial y}\right)^{\alpha}\right)
\end{aligned}
$$

(see Theorem 6.7 in Chapter I of Kumano-go [6]). For $p(y, \eta, \tau)$ $\in S_{\rho, \delta}^{m_{1}}, q(y, \eta, \tau) \in S_{\rho, \delta}^{m_{2}}$ set

$$
\sigma(p \circ q)(y, \eta, \tau)=\text { Os- } \iint e^{-i y^{\prime} \eta^{\prime}} p\left(y, \eta+\eta^{\prime}, \tau\right) q\left(y+y^{\prime}, \eta, \tau\right) d y^{\prime} d \eta^{\prime} .
$$

Then we have $\sigma(p \circ q)(y, \eta, \tau) \in S_{\rho, \delta}^{m_{1}+m_{2}}$ and

$$
\sigma(p \circ q)\left(y, D_{y}, \tau\right) u\left(y^{\prime}\right)=p\left(y^{\prime}, D_{y}, \tau\right)(q u)\left(y^{\prime}\right), u(y) \in \mathscr{S}
$$

(see Theorem 2.5 in Chapter II of Kumano-go [6]). Moreover the following asymptotic expansion formula is obtained for any integer $N$ $(>0)$ :

$$
\begin{gathered}
\sigma(p \circ q)(y, \eta, \tau)-\sum_{\alpha=0}^{N-1} \frac{1}{\alpha !} \frac{\partial^{\alpha} p}{\partial \eta^{\alpha}}(y, \eta, \tau) D_{y}^{\alpha} q(y, \eta, \tau) \\
=\int_{0}^{1} \frac{(1-\theta)^{N-1}}{(N-1) !}\left\{\text { Os- } \iint e^{-i y^{\prime} \eta^{\prime}} \frac{\partial^{N} p}{\partial \eta^{N}}\left(y, \eta+\theta \eta^{\prime}, \tau\right)\right. \\
\left.\quad \times D_{y}^{N} q\left(y+y^{\prime}, \eta, \tau\right) d y^{\prime} d \eta^{\prime}\right\} d \theta \quad \in S_{\rho, \delta}^{m_{1}+m_{2}-(\rho-\delta) N}
\end{gathered}
$$

(see Theorem 3.1 in Chapter II of Kumano-go [6]).

Now let us prove a lemma used later. The equation (in $\xi$ )

$$
\tau^{2}+\xi^{2}+\eta^{2}=0 \quad\left(\gamma=\operatorname{Re} \tau>0, \eta \in \mathbb{R}^{1}\right)
$$

has a root $\xi_{+}(\eta,-)$ with a positive imaginary part and a one with a 
negative imaginary part. It is easily seen that

$$
\begin{aligned}
& \operatorname{Im} \xi_{+}(\eta, \tau) \geqq \gamma, \\
& \left|\xi_{+}(\eta, \tau)\right| \geqq \delta \gamma^{1 / 2}\left(\eta^{2}+|\tau|^{2}\right)^{1 / 4} \quad(\delta>0), \\
& \xi_{+}(\eta, \tau) \in S_{0,0}^{1} .
\end{aligned}
$$

Furthermore, there is a function $q(\eta, \tau) \in S_{0,0}^{0}$ for positive integers $k, l$ such that

$$
\frac{\partial^{l}}{\partial \eta^{l}}\left\{\xi_{+}(\eta, \tau)^{-k}\right\}=q(\eta, \tau) \xi_{+}(\eta, \tau)^{-k}
$$

For an integer $k(>0)$ and $\psi(y) \in \mathcal{B}^{\infty}$ we set

$$
p_{\theta}(y, \eta, \tau)=\text { Os- } \iint e^{-i y^{\prime} \eta^{\prime}} \psi\left(y+y^{\prime}\right) \xi_{+}\left(\eta+\theta \eta^{\prime}, \tau\right)^{-k} d y^{\prime} d \eta^{\prime}
$$

where the parameter 0 moves on $[0,1]$. Then we have

Lemma 2. 1. (i) $\left\{p_{0}(y, \eta, \tau)\right\}_{0 \leq \theta \leq 1}$ is a bounded set in $S_{0,0}^{-k / 2}$, that is, the estimate

$$
\left|\frac{\partial^{\alpha+\beta}}{\partial y^{\beta} \partial \eta^{\alpha}} p_{\theta}(y, \eta, \tau)\right| \leqq C_{\alpha \beta}\left(\eta^{2}+|\tau|^{2}\right)^{-k / 1}
$$

holds for a constant $C_{\alpha \beta}$ independent of $\theta$ and $\tau(\operatorname{Re} \tau \geqq 1)$.

(ii) $\quad\left\{p_{\theta}(y, \eta, \tau) \xi_{+}(\eta, \tau)\right\}_{0 \leq \Theta \leq 1}$ is a bounded set in $S_{0,0}^{-k / 2+1 / 2}$.

Proof. Noting (2.4) and (2.5), we can prove (i) of the lemma in the same way as in Kumano-go [6] (see Lemma 2.4 in Chapter II of [6]). Let us give the proof only in the case $\alpha=\beta=0$. For a positive integer $m$ we have

$$
\begin{gathered}
p_{\theta}(y, \eta, \tau)=\iint e^{-i y^{\prime} \eta^{\prime}}\left(1+\eta^{\prime 2}\right)^{-m}\left(1+D_{y^{\prime}}^{2}\right)^{m}\left(1+y^{\prime 2}\right)^{-m} \phi\left(y+y^{\prime}\right) \\
\cdot\left(1+D_{\eta^{\prime}}^{2}\right)^{m}\left(\xi_{+}\left(\eta+\theta \eta^{\prime}, \tau\right)^{-k}\right) d y^{\prime} d \eta^{\prime}
\end{gathered}
$$

Obviously this is a $C^{\infty}$ function in $(y, \eta)$. By $(2.5)$ we can write

$$
p_{\theta}(y, \eta, \tau)=\iint \Phi\left(y, y^{\prime}, \eta, \eta^{\prime}, \tau, \theta\right) \xi_{+}\left(\eta+\theta \eta^{\prime}, \tau\right)^{-k} d y^{\prime} d \eta^{\prime}
$$




$$
\begin{aligned}
& =\iint_{\left|\eta^{\prime}\right| \Sigma_{i} ; \mid / 2} \Phi \cdot \xi_{+}\left(\eta+\theta \eta^{\prime}, \tau\right)^{-k} d y^{\prime} d \eta^{\prime} \\
& \quad+\iint_{\left|\eta^{\prime}\right| \geq|\eta| / 2} \Phi \cdot \xi_{+}\left(\eta+\theta \eta^{\prime}, \tau\right)^{-k} d y^{\prime} d \eta^{\prime} \\
& \equiv I_{1}+I_{2},
\end{aligned}
$$

where $\left|\Phi\left(y, y^{\prime}, \eta, \eta^{\prime}, \tau, \theta\right)\right| \leqq C_{1}\left(1+\eta^{\prime 2}\right)^{-m}\left(1+y^{\prime 2}\right)^{-m} \quad\left(C_{1}\right.$ is a constant independent of $y, y^{\prime}, \eta, \eta^{\prime}, \tau$ and $\theta \quad(\gamma=\operatorname{Re} \tau \geqq 1$ and $\left.0 \leqq \theta \leqq 1)\right)$. Since $\frac{1}{2}\left(\eta^{2}+|\tau|^{2}\right)^{1 / 2} \leqq\left(\left(\eta+\theta \eta^{\prime}\right)^{2}+|\tau|^{2}\right)^{1 / 2} \leqq \frac{3}{2}\left(\eta^{2}+|\tau|^{2}\right)^{1 / 2}$ when $\left|\eta^{\prime}\right| \leqq \frac{|\eta|}{2}$ and 0 $\leqq \theta \leqq 1$, it follows from (2.4) that

$$
\begin{gathered}
\left|I_{1}\right| \leqq C_{2} \iint\left(1+\eta^{\prime 2}\right)^{-m}\left(1+y^{\prime 2}\right)^{-m} d y^{\prime} d \eta^{\prime}\left(\eta^{2}+|\tau|^{2}\right)^{-(k / 4)} \\
(\gamma=\operatorname{Re} \tau \geqq 1) .
\end{gathered}
$$

Noting that $\left|\xi_{+}\left(\eta+\theta \eta^{\prime}, \tau\right)^{-k}\right| \leqq C|\tau|^{-k / 2}$, we have

$$
\begin{gathered}
\left|I_{2}\right| \leqq C_{3} \iint_{\left|\eta^{\prime}\right| \geq|\eta| / 2}\left(\eta^{2}+|\tau|^{2}\right)^{k / 4}\left(1+\eta^{\prime 2}\right)^{-m}\left(1+y^{\prime 2}\right)^{-m}|\tau|^{-k / 2} \\
\times d y^{\prime} d \eta^{\prime}\left(\eta^{2}+|\tau|^{2}\right)^{-k / 4} \\
\leqq C_{4} \iint\left(1+y^{\prime 2}\right)^{-m}\left(1+\eta^{\prime 2}\right)^{-m+k / 2} d y^{\prime} d \eta^{\prime}\left(\eta^{2}+|\tau|^{2}\right)^{-k / 4} \\
(\gamma=\operatorname{Re} \tau \geqq 1) .
\end{gathered}
$$

Therefore the estimate $\left|p_{\theta}(y, \eta, \tau)\right| \leqq C_{5}\left(\eta^{2}+|\tau|^{2}\right)^{-k / 4}$ is obtained.

Next let us show (ii) of the lemma. By Taylor's expansion

$$
\xi_{+}(\eta, \tau)=\xi_{+}\left(\eta+\theta \eta^{\prime}, \tau\right)+\int_{0}^{1} \frac{\partial \xi_{+}}{\partial \eta}\left(\eta+(1-\mu) \theta \eta^{\prime}, \tau\right) d \mu\left(-\theta \eta^{\prime}\right)
$$

we have

$$
\begin{aligned}
& p_{\theta}(y, \eta, \tau) \xi_{+}(\eta, \tau)=\text { Os- } \iint e^{-i y^{\prime} \eta^{\prime}} \psi\left(y+y^{\prime}\right) \xi_{+}\left(\eta+\theta \eta^{\prime}, \tau\right)^{-k+1} d y^{\prime} d \eta^{\prime} \\
& \quad-\text { Os- } \iint e^{-i y^{\prime} \eta^{\prime}} \psi\left(y+y^{\prime}\right) \xi_{+}\left(\eta+\theta \eta^{\prime}, \tau\right)^{-k} \\
& \quad \times\left\{\int_{0}^{1} \frac{\partial \xi_{+}}{\partial \eta}\left(\eta+(1-\mu) \theta \eta^{\prime}, \tau\right) d \mu\right\} \theta \eta^{\prime} d y^{\prime} d \eta^{\prime}
\end{aligned}
$$

From (i) of the lemma, the first term belongs to $S_{0,0}^{-k / 2+1 / 2}$ and is bounded there when $0 \leqq \theta \leqq 1$. Since $\frac{\partial \xi_{+}}{\partial \eta}(\eta, \tau)=-\frac{\eta}{\xi_{+}(\eta, \tau)}$, the second term is 
of the form

$$
\begin{aligned}
& \text { Os- } \iint e^{-i y^{\prime} \eta^{\prime}} \psi\left(y+y^{\prime}\right) \xi_{+}\left(\eta+\theta \eta^{\prime}, \tau\right)^{-k} \\
& \quad \times\left\{\int_{0}^{1} \xi_{+}\left(\eta+\theta(1-\mu) \eta^{\prime}, \tau\right)^{-1} d \mu\right\} \eta^{\prime} d y^{\prime} d \eta^{\prime} \theta \eta \\
& + \text { Os- } \iint e^{-i y^{\prime} \eta^{\prime}} \psi\left(y+y^{\prime}\right) \xi_{+}\left(\eta+\theta \eta^{\prime}, \tau\right)^{-k} \\
& \quad \times\left\{\int_{0}^{1} \xi_{+}\left(\eta+\theta(1-\mu) \eta^{\prime}, \tau\right)^{-1}(1-\mu) d \mu\right\} \eta^{\prime 2} d y^{\prime} d \eta^{\prime} \theta^{2} \\
& \equiv J_{1} \theta \eta+J_{2} \theta^{2}
\end{aligned}
$$

In the same way as in (i) of the lemma, we see that $J_{1}$ and $J_{2}$ belong to $S_{0,0}^{-k / 2-1 / 2}$ boundedly when $0 \leqq \theta \leqq 1$. Therefore (ii) of the lemma is obtained.

\section{§3. Existence of the Solution}

Let $H_{m}(M)$ be the Sobolev space on $M$ of order $m$ and $\|\cdot\|_{m, Y}$ its norm. We denote by $H_{m, r}\left(\boldsymbol{R}_{+}^{2} \times \mathbb{R}_{+}^{1}\right) \quad(\gamma>0, m=0,1,2, \cdots)$ the functional space $\left\{u(x, y, t): e^{-r t} u \in H_{m}\left(\boldsymbol{R}_{+}^{2} \times \mathbb{R}_{+}^{1}\right)\right\}$ with the norm

$$
|u|_{m, \tau}^{2}=\sum_{i+j+k+l=m} \int_{\boldsymbol{R}_{+}^{2} \times \boldsymbol{R}_{+}^{1}}\left|e^{-\gamma t} \gamma^{i} D_{t}^{j} D_{x}^{k} D_{y}^{l} u\right|^{2} d x d y d t .
$$

In the same way, we define the space $H_{m, r}\left(\mathbb{R}^{1} \times \mathbb{R}_{+}^{1}\right)$ and its norm $\langle\cdot\rangle_{m, r}$. Let us define the norm $[\cdot]_{m, r}(m=0,1, \cdots)$ of $H_{m}\left(\mathbb{R}_{+}^{2}\right)\left(H_{m}\left(\mathbb{R}^{2}\right)\right)$ by $[v]_{m, r}^{2}=\sum_{i+j+k=m} \int\left|\gamma^{i} D_{x}^{j} D_{y}^{k} v(x, y)\right|^{2} d x d y$.

In this section we consider the mixed problem

$$
\left\{\begin{array}{l}
\square u(x, y, t)=f(x, y, t) \quad \text { in } \mathbb{R}_{+}^{2} \times(0, \infty), \\
\left.\left(\frac{\partial}{\partial y}+\varphi(y)^{2} \frac{\partial}{\partial x}\right) u\right|_{x=0}=g(y, t) \quad \text { on } \mathbb{R}^{1} \times(0, \infty), \\
\left.\frac{\partial u}{\partial t}\right|_{t=0}=u_{1}(x, y) \text { on } \mathbb{R}_{+}^{2}, \\
\left.u\right|_{t=0}=u_{0}(x, y) \text { on } \mathbb{R}_{+}^{2} .
\end{array}\right.
$$

Assume that $\varphi(y)\left(\in \mathscr{B}^{\infty}\right)$ is real-valued and satisfies $\inf _{|y| \geqq y_{0}}|\varphi(y)|>0$ for a large constant $y_{0}$. Then we have 
Theorem 3. 臬. There exists a constant $\gamma_{m}(>0)$ for $m=2,3, \cdots$ such that if $r \geqq \gamma_{m}$ the problem (3.1) has a unique solution $u(x, y, t)$ in $H_{m, r}\left(\mathbb{R}_{+}^{2} \times \mathbb{R}_{+}^{1}\right) \quad$ for any $\left(u_{0}, u_{1}, f, g\right) \in H_{m+4}\left(\mathbb{R}_{+}^{2}\right) \times H_{m+3}\left(\mathbb{R}_{+}^{2}\right)$ $\times H_{m+3, r}\left(\mathbb{R}_{+}^{2} \times \mathbb{R}_{+}^{1}\right) \times H_{m+3, r}\left(\mathbb{R}^{1} \times \mathbb{R}_{+}^{1}\right)$ satisfying the compatibility condition of order $m+2$. Furthermore, the following estimate for this solution holds:

$$
\gamma^{2}|u|_{m, r}^{2} \leqq C\left(|f|_{m+3, r}^{2}+\langle g\rangle_{m+3, r}^{2}+\gamma\left[u_{0}\right]_{m+4, r}^{2}+\gamma\left[u_{1}\right]_{m+3, r}^{2}\right) .
$$

The discussion in this section is applicable to the problem with the boundary operator $\frac{\partial}{\partial y}-\varphi(y)^{2} \frac{\partial}{\partial x}$ instead of $\frac{\partial}{\partial y}+\varphi(y)^{2} \frac{\partial}{\partial x}$.

Combining the following corollary of Theorem 3.1 with the result in $\S 4$ concerning the domain of dependence, we can prove easily Theorem stated in Introduction.

Corollary of Theorem 3.1. Let the assumptions of Theorem in Introduction be satisfied. Then, the problem (1.1) has a unique solution $u(x, y, t)$ in $H_{m}\left(\boldsymbol{R}_{+}^{2} \times(0, T)\right) \quad(m=6,7, \cdots ; T<\infty)$ for any $\left(u_{0}(x, y), u_{1}(x, y), f(x, y, t), g(y, t)\right) \in H_{m+4}\left(\mathbb{R}_{+}^{2}\right) \times H_{m+3}\left(\mathbb{R}_{+}^{2}\right) \times H_{m+3}\left(\mathbb{R}_{+}^{2}\right.$ $\times(0, T)) \times H_{m+3}\left(R^{1} \times(0, T)\right)$ satisfying the compatibility condition of order $m+2$.

Proof of Corollary. Extending $f(x, y, t)$ and $g(y, t)$ in the data to $t>T$ continuously, we obtain a solution $u(x, y, t) \in H_{m}\left(R_{+}^{2} \times(0, T)\right)$ of (1. 1) by means of Theorem 3.1.

Let us show the uniqueness of the solution. Let $\widetilde{u}(x, y, t) \in H_{6}\left(\boldsymbol{R}_{+}^{2}\right.$ $\times(0, T))$ be a solution of (1.1) for zero data. For any $\varepsilon>0$ choose a function $\chi(t) \in C^{\infty}$ such that $\chi(t)=1$ for $0 \leqq t \leqq T-\varepsilon$ and $\chi(t)=0$ for $t \geqq T$. Then $w=\chi \widetilde{u}$ is a solution of the equation

$$
\begin{cases}\square w(x, y, t)=[\square, \chi] \widetilde{u}(x, y, t) \text { in } \mathbb{R}_{+}^{2} \times(0, \infty), \\ B w(y, t)=0 & \text { on } \mathbb{R}^{1} \times(0, \infty), \\ \frac{\partial w}{\partial t}{ }_{t=0}=0 & \text { on } \mathbb{R}_{+}^{2}, \\ \left.w\right|_{t=0}=0 & \text { on } \mathbb{R}_{+}^{2},\end{cases}
$$


where $[\square, \chi]$ is the commutator of $\square$ and $\chi . \quad[\square, \chi] \widetilde{u}$ belongs to $H_{5, y}\left(\mathbb{R}_{+}^{2}\right.$ $\times \mathbb{R}_{+}^{1}$ ) and its support lies in $\{T-\varepsilon \leqq t \leqq T\}$. Obviously the result in Theorem 3.1 is valid also when the initial condition is posed on $t=T$ - $\varepsilon$. Therefore there is a solution of (3.2) in $H_{2, r}\left(\mathbb{R}_{+}^{2} \times \mathbb{R}_{+}^{1}\right)$ whose support lies in $\{T-\varepsilon \leqq t<\infty\}$. From the uniqueness of the solution of (3. 1), we conclude $\operatorname{supp}[\chi \widetilde{u}] \subset\{T-\varepsilon \leqq t<\infty\}$. Since $\varepsilon$ is any positive constant, $\widetilde{u}$ is equal to zero in $\{0<t<T\}$. The proof is complete.

From now on, we shall prove Theorem 3.1.

1) At first we reduce the problem to the equation on the boundary, as is stated in Introduction. Tsuji in [8] also employed the same reduction. Let us define

$$
\begin{aligned}
& \stackrel{+}{H}_{n^{\prime}, 2}\left(\mathbb{R}_{i}^{2}<\mathbb{R}^{1}\right)=\left\{u\left(r, u^{\prime}, \iota\right) \subseteq H_{n}\left(\mathbb{R}_{+}^{2} \times \mathbb{R}^{1}\right) ; \operatorname{supp}[u] こ\{t \geqq 0\},\right. \\
& \left.|u|_{m \cdot r}<\infty\right\} \quad(\gamma>0, m=0,1, \cdots) \text {, }
\end{aligned}
$$

and $\stackrel{+}{H}_{m, r}\left(\mathbb{R}^{1} \times \mathbb{R}^{1}\right)$ in the same way. The following two propositions are well known.

Proposition 3.1 . We have a unique solution $u^{\prime}\left(x, \mathrm{y}^{\prime}, t\right)$ in $H_{m+1, r}\left(\mathbb{R}^{2} \times \mathbb{R}_{+}^{1}\right) \quad(\gamma \geq 1, m=2,3, \cdots)$ satisfying the Cauchy problem

$$
\begin{cases}\square u^{\prime}(x, y, t)=f^{\prime}(x, y, t) & \text { in } \mathbb{R}^{2} \times \mathbb{R}^{2}+, \\ \frac{\partial u^{\prime}}{\partial t}{ }_{t=0}=u_{1}^{\prime}(x, y) & \text { on } \mathbb{R}^{2}, \\ u^{\prime}{ }_{t=0}=u_{0}^{\prime}(x, y) & \text { on } \mathbb{R}^{2}\end{cases}
$$

for any $\left(f^{\prime}, u_{0}^{\prime}, u_{1}^{\prime}\right) \in H_{m, r}\left(\mathbb{R}^{2} \times \mathbb{R}_{+}^{1}\right) \times H_{m+1}\left(\mathbb{R}^{2}\right) \times H_{m}\left(\mathbb{R}^{2}\right)$, and have the estimatc

$$
\gamma^{2}\left|u^{\prime}\right|_{m+1, r}^{2} \leqq C\left(\left|f^{\prime}\right|_{m, r}^{2}+\gamma\left[u_{0}^{\prime}\right]_{m+1, r}^{2}+\gamma\left[u_{1}^{\prime}\right]_{r, r}^{2}\right)
$$

Proposition 3.2. For any $h(y, t) \in \stackrel{+}{H}_{m, r}\left(\mathbb{R}^{1} \times \mathbb{R}^{1}\right)(\gamma \geqq 1, m=2,3$, ․) there exists a solution $r(x, y, l) \in \stackrel{+}{H}_{m, 7}\left(\mathbb{R}_{+}^{2} \times \mathbb{R}^{1}\right)$ of the Dirichlet problem

$$
\left\{\begin{array}{lll}
\square w(x, y, t)=0 & \text { in } & \mathbb{R}_{+}^{2} \times \mathbb{R}^{1} \\
\left.w\right|_{x=0}=h\left(\jmath^{\prime}, t\right) & \text { on } & \mathbb{R}^{1} \times \mathbb{R}^{1}
\end{array}\right.
$$


This solution is unique in $\stackrel{+}{H}_{2, r}\left(\boldsymbol{R}_{+}^{2} \times \boldsymbol{R}^{1}\right)$ and the following estimate holds:

$$
|w|_{m, r}^{2} \leqq \frac{C}{\gamma}\langle h\rangle_{m, r}^{2}
$$

We set for $h(y, t) \in \stackrel{+}{H}_{m, r}\left(\boldsymbol{R}^{1} \times \boldsymbol{R}^{1}\right)$

$$
T h=B w\left(=\left.\left(\frac{\partial}{\partial y}+\varphi(y)^{2} \frac{\partial}{\partial x}\right) w\right|_{x=0}\right),
$$

where $w$ is the solution stated in Proposition 3.2. Let $\left(u_{0}, u_{1}, f, g\right)$ be the data in Theorem 3.1. Extend $u_{0}(x, y), u_{1}(x, y)$ and $f(x, y, t)$ to $x<0$ continuously, and denote them by $u_{0}^{\prime}(x, y), u_{1}^{\prime}(x, y)$ and $f^{\prime}(x, y, t)$ respectively. For these $u_{0}^{\prime}, u_{1}^{\prime}, f^{\prime}$ we have the solution $u^{\prime} \in H_{m+4}\left(\boldsymbol{R}^{2} \times \boldsymbol{R}_{+}^{1}\right)$ stated in Proposition 3.1. Define $g^{\prime}(y, t)=g(y, t)-B u^{\prime}(y, t)$ for $t>0$ and $g^{\prime}(y, t)=0$ for $t<0$. Then, noting that the data $\left(u_{0}, u_{1}, f, g\right)$ satisfy the compatibility condition of order $m+2$, we see that $g^{\prime}(y, t)$ belongs to $\stackrel{+}{H}_{m+2, r}\left(\mathbb{R}^{1} \times \mathbb{R}^{1}\right)$. If the equation $T h^{\prime}=g^{\prime}$ has a solution $h^{\prime}(y, t)$ $\in \stackrel{+}{H}_{m, r}\left(\mathbb{R}^{1} \times \mathbb{R}^{1}\right)$, then $u(x, y, t)=u^{\prime}(x, y, t)+w^{\prime}(x, y, t) \quad\left(w^{\prime}\right.$ is the solution of (3.3) for $\left.h=h^{\prime}\right)$ belongs to $H_{m, r}\left(\boldsymbol{R}_{+}^{2} \times \boldsymbol{R}_{+}^{1}\right)$ and satisfies (3.1). So we have only to investigate the equation $T h=g$.

Lemma 3. 1. There is a constant $\gamma_{m}$ for $m(\geqq 0)$ such that if $\gamma \geqq \gamma_{m}$ we have a unique solution $h$ of $T h=g$ in $\stackrel{+}{H}_{m, r}\left(\boldsymbol{R}^{1} \times \boldsymbol{R}^{1}\right)$ for any $g \in \stackrel{+}{H}_{m+2, r}\left(\mathbb{R}^{1} \times \mathbb{R}^{1}\right)$ and have the estimate

$$
\langle h\rangle_{m, r}^{2} \leqq \frac{C}{r}\langle T h\rangle_{m+2, r}^{2} .
$$

It is easy to see that Lemma 3.1, Proposition 3.1 and Proposition 3. 2 yield Theorem 3. 1 .

2) Let us prove Lemma 3.1. We set

$$
P=D_{y}+\varphi(y)^{2} \xi_{+}\left(D_{y}, \tau\right) \quad(\tau=\gamma+i \sigma)
$$

where $\xi_{T}(\eta, \tau)$ is the root stated in $\S 2$. We define the Fourier-Laplace transform of $h(y, t) \in \stackrel{+}{H}_{m, r}\left(\boldsymbol{R}^{1} \times \boldsymbol{R}^{1}\right)$ by 


$$
\begin{aligned}
\tilde{h}(\eta, \tau) & =\mathscr{I}_{(y, t) \rightarrow(\eta, \sigma)}\left[e^{-\gamma t} h(y, t)\right] \quad(\tau=\gamma+i \sigma) \\
& =\int e^{-\tau t-i y \eta} h(y, t) d y d t .
\end{aligned}
$$

Since the solution $v(x, y, t)$ in Proposition 3.2 is expressed by the form

$$
w(x, y, t)=\int \exp \left(t \tau+i y \eta+i x \xi_{+}(\eta, \tau)\right) \tilde{h}(\eta, \tau) d \eta d \sigma,
$$

$T$ is of the form

$$
T h(y, t)=i \int e^{t \tau+i y \eta}\left(\eta+\varphi(y)^{2} \xi_{+}(\eta, \tau)\right) \tilde{h}(\eta, \tau) d \eta d \sigma
$$

Noting that $\eta+\varphi(y)^{2} \xi_{+}(\eta, \tau)$ is the symbol of $P$, we see that Lemma 3.1 is derived from the following lemma.

Lemma 3. 2. We have for any $s \in \mathbb{R}$

(i) $\quad\|P u\|_{s+2}^{2} \geqq C_{1}\left(\gamma-\gamma_{1}\right)\|u\|_{s}^{2}, \quad u(y) \in \mathscr{S}\left(\mathrm{K}^{1}{ }^{1}\right)$.

(ii) $\quad\left\|P^{*} u\right\|_{s+2}^{2} \geqq C_{2}\left(\gamma-\tau_{2}\right)\|u\|_{s}^{2}, \quad u \in \mathscr{S}$,

where $P^{*}$ is the formally adjoint operator of $P$ and the constants $C_{1}, C_{2}, \gamma_{1}, \gamma_{2}$ do not depend on $\tau=\gamma+i \sigma(\gamma \geq 1)$. (\|l| $\|_{s}$ is the norm defined in \$2).

The following lemma plays an essential role for the proof of Lemma 3. 2 .

Lemma 3. 3. Let $\psi(\nu) \in \mathscr{B}^{\infty}\left(\mathbb{R}^{1}\right)$. There exist symbols $\alpha(y, \eta, \tau)$, $\beta(y, \eta, \tau) \in S_{0,0}^{-1 / 2}$ such that

$$
\begin{aligned}
& {\left[\psi, \xi_{+}\right]=\alpha D_{y}+\beta,} \\
& \alpha(y, \eta, \tau) \xi_{+}(\eta, \tau), \quad \beta(y, \eta, \tau) \xi_{+}(\eta, \tau) \in S_{0,0}^{0},
\end{aligned}
$$

where $\left[\psi, \xi_{+}\right]=\psi^{\prime} \xi_{+}-\xi_{+} \psi . \quad$ (This statement is valid for $\left.\overline{\xi_{+}(\eta, \tau)}\right)$.

Proof. By means of the asymptotic expansion formula (2.2). the symbol of $\left[\psi, \xi_{+}\right]$is expressed by the form 


$$
-\int_{0}^{1}\left\{\operatorname{Os}_{-} \iint e^{-i y^{\prime} \eta^{\prime}}\left(D_{y} \psi\right)\left(y+y^{\prime}\right) \frac{\partial \xi_{+}}{\partial \eta}\left(\eta+\theta \eta^{\prime}, \tau\right) d y^{\prime} d \eta^{\prime}\right\} d \theta \text {. }
$$

Since $\frac{\partial}{\partial \eta} \xi_{+}(\eta, \tau)=-\frac{\eta}{\xi_{+}(\eta, \tau)}$, it follows that

$$
\begin{aligned}
\text { Os- } \iint e^{-i y^{\prime} \eta^{\prime}}\left(D_{y} \psi\right)\left(y+y^{\prime}\right) \frac{\partial \xi_{+}}{\partial \eta}\left(\eta+\theta \eta^{\prime}, \tau\right) d y^{\prime} d \eta^{\prime} \\
=- \text { Os- } \iint e^{-i y^{\prime} \eta^{\prime}}\left(D_{y} \psi\right)\left(y+y^{\prime}\right) \cdot \xi_{+}\left(\eta+\theta \eta^{\prime}, \tau\right)^{-1} d y^{\prime} d \eta^{\prime} \cdot \eta \\
\quad-\text { Os- } \iint e^{-i y^{\prime} \eta^{\prime}}\left(D_{y}^{2} \psi\right)\left(y+y^{\prime}\right) \cdot \xi_{+}\left(\eta+\theta \eta^{\prime}, \tau\right)^{-1} d y^{\prime} d \eta^{\prime} \cdot \theta \\
\equiv \alpha_{0}(y, \eta, \tau) \eta+\beta_{\theta}(y, \eta, \tau) \theta .
\end{aligned}
$$

Here we have used the formula (2.1). Lemma 2.1 implies that $\left\{\alpha_{\theta}^{\prime}(y, \eta, \tau)\right\}_{0 \leqq \theta \leqq 1},\left\{\beta_{\theta}(y, \eta, \tau)\right\}_{0 \leqq \theta \leqq 1}$ and $\left\{\alpha_{\theta}(y, \eta, \tau) \xi_{+}(\eta, \tau)\right\}_{0 \leqq \theta \leqq 1}, \quad\left\{\beta_{\theta}(y\right.$, $\left.\eta, \tau) \xi_{+}(\eta, \tau)\right\}_{0 \leqq \theta \leqq 1}$ are bounded in $S_{0,0}^{-1 / 2}$ and $S_{0,0}^{0}$ respectively. Therefore, setting

$$
\alpha(y, \eta, \tau)=\int_{0}^{1} \alpha_{\theta}(y, \eta, \tau) d \theta, \quad \beta(y, \eta, \tau)=\int_{0}^{1} \beta_{\theta}(y, \eta, \tau) \theta d \theta,
$$

we get the lemma. The proof is complete.

Let $A^{s}(s \in \mathbb{R})$ be the operator with the symbol $\left(\eta^{2}+|\tau|^{2}\right)^{s / 2}$. We obtain the following lemma by an easier argument than the proof of Lemma 3. 3 .

Lemma 3. 4. Let $\psi(y) \in \mathscr{B}^{\infty}\left(\boldsymbol{R}^{1}\right)$. There exist symbols $a_{s-2}(y, \eta, \tau), b_{s-2}(y, \eta, \tau) \in S_{1,0}^{s-2}(s \in \mathbb{R})$ such that

$$
\left[\psi, \Lambda^{s}\right]=a_{s-2} D_{y}+b_{s-2} .
$$

From the assumption $\inf _{|y| \geqq y_{0}}|\varphi(y)|>0$, we get

Lemma 3.5. For $s \in \mathbb{R}$ we have

$$
\|u\|_{s} \leqq C\left(\|P u\|_{s}+\left\|\varphi \xi_{+} u\right\|_{s}+\frac{1}{\gamma}\|u\|_{s}\right), \quad u \in \mathscr{S},
$$

where the constant $C$ does not depend on $\tau(\gamma=\operatorname{Re} \tau \geqq 1)$. 
Proof. Let $\chi(y)\left(\in \mathcal{B}^{\infty}\right)=1$ for $|y| \leqq y_{0}, \chi(y)=0$ for $|y| \geqq y_{0}+1$ and $0 \leqq \chi(y) \leqq 1$. Then it follows from $\inf _{|y| \geqq y_{0}}|\varphi(y)|>0$ that

$$
\|(1-\chi) v\|_{0} \leqq C_{1}\|\varphi v\|_{0}, \quad\left\|\left(D_{y} \chi\right) v\right\|_{0} \leqq C_{2}\|\varphi v\|_{0} .
$$

By Poincaré's inequality we have

$$
\begin{aligned}
\|u\|_{s} & \leqq C_{3}\left(\left\|D_{y}\left(\chi \Lambda^{s} u\right)\right\|_{0}+\left\|(1-\chi) \Lambda^{s} u\right\|_{0}\right) \\
& \leqq C_{4}\left(\left\|D_{y} u\right\|_{s}+\left\|\left(D_{y} \chi\right) \Lambda^{s} u\right\|_{0}+\left\|(1-\gamma) \Lambda^{s} u\right\|_{0}\right) .
\end{aligned}
$$

Noting that $D_{y}=P-\varphi^{2} \xi_{+}$, we get

$$
\|u\|_{s} \leqq C_{5}\left(\|P u\|_{s}+\left\|\varphi \xi_{+} u \iota\right\|_{s}+\left\|\varphi \lambda^{s} u\right\|_{0}\right) .
$$

By (2.3) and Lemma 3. 3, we have

$$
\begin{aligned}
\left\|\varphi \Lambda^{s} u\right\|_{0} & \leqq \frac{C_{6}}{\gamma}\left\|\xi_{+} \varphi \Lambda^{s} u\right\|_{0} \\
& \leqq \frac{C_{6}}{\gamma}\left(\left\|\xi_{+}\left[\varphi, \Lambda^{s}\right] u\right\|_{0}+\left\|\varphi \xi_{+} u\right\|_{s}+\left\|\left[\varphi, \xi_{+}\right] u\right\|_{s}\right) \\
& \leqq \frac{C_{7}}{\gamma}\left(\|u\|_{s}+\left\|\varphi \xi_{+} u\right\|_{s}+\|P u\|_{s}\right)
\end{aligned}
$$

Therefore the lemma is obtained.

Proof of Lemma 3. 2. Noting that $P^{*} u=D_{y} u+\bar{\xi}_{1} \varphi^{2} u$, we can obtain the estimate for $P^{*}$ in the same way as for $P$. So let us prove only (i) of the lemma. This is derived from the inequality

(3. 4) $\quad \operatorname{Im}\left(\Lambda^{s} \xi_{+} P u, \Lambda^{s} \xi_{+} u\right) \geqq\left(\gamma-\gamma_{1}\right)\left\|\varphi \xi_{+} u\right\|_{s}^{2}-C_{1}\left(\|P u\|_{s}^{2}+\|u\|_{s}^{2}\right)$,

where the constants $\gamma_{1}, C_{1}$ do not depend on $\tau$. In fact, combining this inequality and Lemma 3.5, we get

$$
\|P u\|_{s+2}\|u\|_{s} \geqq\left(\gamma-\gamma_{1}\right) C_{2}\|u\|_{s}^{2}-C_{3}\left(\|P u\|_{s+1 / 2}^{2}+\|u\|_{s}^{2}\right),
$$

which proves (i) of the lemma.

In view of (2.3) we have

$$
\begin{aligned}
\operatorname{Im}(P u, u) & =\operatorname{Im}\left\{\left(D_{y} u, u\right)+\left(\xi_{+} \varphi u, \varphi u\right)+\left(\left[\varphi, \xi_{+}\right] u, \varphi u\right)\right\} \\
& \geqq \gamma\|\varphi u\|_{0}^{2}-\left|\left(\left[\varphi, \xi_{+}\right] u, \varphi u\right)\right| .
\end{aligned}
$$

Therefore, 
$\operatorname{Im}\left(P \Lambda^{s} \xi_{+} u, \Lambda^{s} \xi_{+} u\right) \geqq \gamma\left\|\varphi \Lambda^{s} \xi_{+} u\right\|_{0}^{2}-\left|\left(\left[\varphi, \xi_{+}\right] \xi_{+} \Lambda^{s} u, \varphi \Lambda^{s} \xi_{+} u\right)\right|$.

Since we can write

$$
\begin{aligned}
{\left[P, \Lambda^{s} \xi_{+}\right] } & =\varphi\left[\varphi, \Lambda^{s} \xi_{+}\right] \xi_{+}+\left[\varphi, \Lambda^{s} \xi_{+}\right] \varphi \xi_{+} \\
& =\varphi\left[\varphi, \xi_{+}\right] \Lambda^{s} \xi_{+}+\varphi \xi_{+}\left[\varphi, \Lambda^{s}\right] \xi_{+}+\left[\varphi, \xi_{+}\right] \Lambda^{s} \varphi \xi_{+}+\xi_{+}\left[\varphi, \Lambda^{s}\right] \varphi \xi_{+},
\end{aligned}
$$

we get

$$
\begin{aligned}
\operatorname{Im}\left(\Lambda^{s} \xi_{+}\right. & \left.P u, \Lambda^{s} \xi_{+} u\right) \geqq r\left\|\varphi \Lambda^{s} \xi_{+} u\right\|_{0}^{2} \\
& -\left\{2\left|\left(\left[\varphi, \xi_{+}\right] \Lambda^{s} \xi_{+} u, \varphi \Lambda^{s} \xi_{+} u\right)\right|+\left|\left(\xi_{+}\left[\varphi, \Lambda^{s}\right] \xi_{+} u, \varphi \Lambda^{s} \xi_{+} u\right)\right|\right. \\
& \left.+\left|\left(\Lambda^{s} \varphi \xi_{+} u,\left[\varphi, \bar{\xi}_{+}\right] \Lambda^{s} \xi_{+} u\right)\right|+\left|\left(\Lambda^{s} \varphi \xi_{+} u, \Lambda^{-s}\left[\varphi, \Lambda^{s}\right] \bar{\xi}_{+} \xi_{+} \Lambda^{s} u\right)\right|\right\} \\
\equiv & \gamma\left\|\varphi \Lambda^{s} \xi_{+} u\right\|_{0}^{2}-\left\{2 I_{1}+I_{2}+I_{3}+I_{4}\right\} .
\end{aligned}
$$

Let us show

$$
I_{i} \leqq C_{4}\left(\|P u\|_{s}^{2}+\left\|\varphi \xi_{+} u\right\|_{s}^{2}+\|u\|_{s}^{2}\right), \quad(i=1,2,3,4) .
$$

By Lemma 3.3 there are symbols $a_{s}(y, \eta, \tau), b_{s}(y, \eta, \tau) \in S_{0,0}^{s}$ such that

$$
\left[\varphi, \xi_{+}\right] \Lambda^{s} \xi_{+}=a_{s} D_{y}+b_{s}\left(=a_{s} P-a_{s} \varphi^{2} \xi_{+}+b_{s}\right) \text {. }
$$

Similarly, $\left[\varphi, \bar{\xi}_{+}\right] \Lambda^{s} \xi_{+}\left(=\left[\varphi, \bar{\xi}_{+}\right] \Lambda^{s} \bar{\xi}_{+} \cdot \bar{\xi}_{+}^{-1} \xi_{+}\right)$is of the same form. Furthermore, from Lemma 3.4 it is easily seen that $\xi_{+}\left[\varphi, \Lambda^{s}\right] \xi_{+}$and $\Lambda^{-s}\left[\varphi, \Lambda^{s}\right] \bar{\xi}_{+} \xi_{+} \Lambda^{s}$ are also of the same form. These facts yield

$$
\begin{aligned}
& I_{i} \leqq C_{5}\left(\|P u\|_{s}^{2}+\left\|\varphi \lambda^{s} \xi_{-} u\right\|_{0}^{2}+\left\|\varphi \xi_{+} u\right\|_{s}^{2}+\|u\|_{s}^{2}\right) \\
& \quad \leqq C_{8}\left(\|P u\|_{s}^{2}+\left\|\varphi \xi_{+} u\right\|_{s}^{2}+\|u\|_{s}^{2}\right) \quad(i=1,2,3,4) .
\end{aligned}
$$

Hence,

$$
\operatorname{Im}\left(\Lambda^{s} \xi_{+} P u, \Lambda^{s} \xi_{+} u\right) \geqq \gamma\left\|\varphi \Lambda^{s} \xi_{+} u\right\|_{0}^{2}-C_{7}\left(\|P u\|_{s}^{2}+\left\|\varphi \xi_{+} u\right\|_{s}^{2}+\|u\|_{s}^{2}\right) .
$$

Noting Lemma 3.4 and $D_{y}=P-\varphi^{2} \xi_{+}$, we have

$$
\left\|\varphi \xi_{+} u\right\|_{s} \leqq\left\|\varphi \Lambda^{s} \xi_{+} u\right\|_{0}+C_{8}\left(\|P u\|_{s-1}+\left\|\varphi \xi_{+} u\right\|_{s-1}+\|u\|_{s-1}\right) .
$$

Therefore, (3.4) is obtained. The proof is complete.

\section{§4. The Domain of Dependence}

In this section we shall study boundedness of the domain of dependence for the problem (1.1). Let the boundary operator in (1.1) be 
of the form $\frac{\partial}{\partial y}+\varphi(y)^{2} \frac{\partial}{\partial x}$ (or $\frac{\partial}{\partial y}-\varphi(y)^{2} \frac{\partial}{\partial x}$ ) and $\varphi(y)$ satisfy the assumptions of Theorem stated in Introduction. Moreover, for simplicity we suppose that $\varphi(0)=0$ and $\varphi(y) \neq 0$ for $y \neq 0$. This is not essential, and boundedness of the domain of dependence holds without this hypothesis. By Ikawa [2] we see that the propagation speed of the solution is finite at the points where $\varphi(y)$ does not vanish. So we examine the domain of dependence near $(x, y)=(0,0)$.

Set

$$
\theta(y)=\int_{0}^{y} \frac{\varphi(s)^{2}}{\left(1+\varphi(s)^{4}\right)^{1 / 2}} d s
$$

Then $\theta(y)$ is an increasing $C^{\infty}$ function and $\left(\frac{d \theta}{d y}\left(y_{0}\right)\right)^{-1}\left(y_{0} \neq 0\right)$ equals the propagation speed for the problem with the frozen boundary condition $\left.\left(\frac{\partial u}{\partial y}+\varphi\left(y_{0}\right)^{2} \frac{\partial u}{\partial x}\right)\right|_{x=0}=g(y, t)$ (cf. Appendix of [2]). We set for $t_{0}(>0)$,

$$
D_{t_{0}}=\left\{(x, y, t): t-t_{0}+\min \left[\theta\left(\sqrt{x^{2}+y^{2}}\right),-\theta\left(-\sqrt{x^{2}+y^{2}}\right)\right]<0\right\} .
$$

Our purpose is to prove

Theorem 4. 1. (i) The problem (1.1) has not a finite propagation speed.

(ii) Let $u(x, y, t) \in H_{6}\left(\mathbb{R}_{+}^{2} \times(0, T)\right)$ be a solution of (1.1) for data $\left(u_{0}, u_{1}, f, g\right) \in H_{7}\left(\mathbb{R}_{+}^{2}\right) \times H_{6}\left(\mathbb{R}_{+}^{2}\right) \times H_{6}\left(\mathbb{R}_{+}^{2} \times(0, T)\right) \times H_{6}\left(\mathbb{R}^{1} \times(0\right.$, T)). If

$$
\left\{\begin{array}{l}
f(x, y, t)=0 \quad \text { on } D_{t_{0}} \cap\left\{x>0,0<t<t_{0}\right\} \\
g(y, t)=0 \quad \text { on } D_{t_{0}} \cap\left\{x=0,0<t<t_{0}\right\} \\
u_{0}(x, y)=0, u_{1}(x, y)=0 \text { on } D_{t_{0}} \cap\{x>0, t=0\}
\end{array}\right.
$$

then $u(x, y, t)=0$ on $D_{t_{0}} \cap\left\{x>0,0<t<t_{0}\right\}$.

Remark 4.1. (ii) of the theorem is valid also when $u(x, y, t)$ $\in C^{\infty}\left(\overline{\boldsymbol{R}_{+}^{2}} \times[0, T]\right)$.

In fact, take a $C^{\infty}$ function $\chi(x, y, t)$ with compact support satisfying $\chi(x, y, t)=1$ in a neighborhood of $\bar{D}_{t_{0}} \cap\left\{x \geqq 0,0 \leqq t \leqq t_{0}\right\}$ and consider 


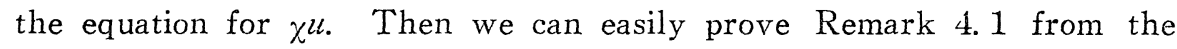
case $u \in H_{\theta}\left(\boldsymbol{R}_{+}^{2} \times(0, T)\right)$.

Proof of Theorem 4.1. At first we prove (ii) of the theorem. Let the data $\left(u_{0}, u_{1}, f, g\right)$ satisfy (4.1). Denote by $\delta(t)$ the inverse of $\theta(y)$ (i.e. $\theta(\delta(t))=t)$. For any $\varepsilon>0$ choose a $C^{\infty}$ function $\varphi_{\varepsilon}(y)$ such that $0 \leqq \varphi_{\varepsilon}(y) \leqq 1, \varphi_{\varepsilon}(0)>0$ and $\operatorname{supp}\left[\varphi_{\varepsilon}\right] \subset\left[-\frac{\delta(\varepsilon)}{2}, \frac{\delta(\varepsilon)}{2}\right]$. We consider the equation

$$
\left\{\begin{array}{l}
\square v(x, y, t)=f(x, y, t) \text { in } \boldsymbol{R}_{+}^{2} \times\left(0, t_{0}-\varepsilon\right), \\
\left.\left(\frac{\partial v}{\partial y}+\left(\varphi(y)^{2}+\varphi_{\varepsilon}(y)\right) \frac{\partial v}{\partial x}\right)\right|_{x=0}=g(y, t) \text { on } \mathbb{R}^{1} \times\left(0, t_{0}-\varepsilon\right), \\
\left.\frac{\partial v}{\partial t}\right|_{t=0}=u_{1}(x, y) \text { on } \boldsymbol{R}_{+}^{2}, \\
\left.v\right|_{t=0}=u_{0}(x, y) \text { on } \mathbb{R}_{+}^{2} .
\end{array}\right.
$$

Since the boundary condition of this equation is non-singular, we can apply the methods of Ikawa [2]. Therefore, there is a solution $v(x, y, t)$ $\left(\in H_{6}\left(\mathbb{R}_{+}^{2} \times\left(0, t_{0}-\varepsilon\right)\right)\right)$ such that

$$
v(x, y, t)=0 \quad \text { on } \quad D_{t_{0}} \cap\left\{x>0,0<t<t_{0}-\varepsilon\right\} .
$$

Obviously this $v(x, y, t)$ satisfies (1. 1) for $0<t<t_{0}-\varepsilon$. From the uniqueness of the solution of (1.1) (see Corollary of Theorem 3.1), we can conclude that $u(x, y, t)=0$ on $D_{t_{0}} \cap\left\{x>0,0<t<t_{0}-\varepsilon\right\}$. Since $\varepsilon$ is any positive constant, (ii) of the theorem is obtained.

Next let us show (i) of the theorem. The idea of the proof is suggested by Kajitani [4] and Appendix of Ikawa [2]. We construct an asymptotic solution

$$
u_{N}(x, y, t)=\sum_{n=0}^{N} e^{i k \varpi(x, y, t)} v_{n}(x, y, t)(i k)^{-n}
$$

in the same way as in [5] such that

$$
\left\{\begin{array}{l}
\square u_{N}(x, y, t)=e^{i k \bullet} \square v_{N}(i k)^{-N} \text { in } \mathbb{R}_{+}^{2} \times[0, T] \quad\left(t_{0}<T\right), \\
B u_{N}(y, t)=0 \text { on } \boldsymbol{R}^{1} \times[0, T] .
\end{array}\right.
$$

By an easy calculation we have 


$$
\begin{aligned}
& e^{-i k t} \square u u_{N}=(i k)^{2}\left(\Phi_{t}^{2}-\Phi_{x}^{2}-\Phi_{y}^{2}\right) \sum v_{n}(i k)^{-n} \\
& \quad+(i k)\left\{(\square \Phi) \sum v_{n}(i k)^{-n}+2 \sum\left(\Phi_{t} \frac{\partial v_{n}}{\partial t}-\Phi_{x^{-}} \frac{\partial v_{n}}{\partial x}-\Phi_{y} \frac{\partial v_{n}}{\partial y}\right)(i k)^{-n}\right\} \\
& \quad+\sum\left(\square v_{n}\right)(i k)^{-n}, \\
& e^{-i k \omega} B u_{N}=(i k)(B \Phi) \sum v_{n}(i k)^{-n}+\sum\left(B v_{n}\right)(i k)^{-n},
\end{aligned}
$$

where $\Phi_{t}, \Phi_{x}, \Phi_{y}$ denote $\frac{\partial \Phi}{\partial t}, \frac{\partial \Phi}{\partial x}, \frac{\partial \Phi}{\partial y}$ respectively. Therefore, we obtain the following equations for the phase function $\Phi(x, y, t)$ and the amplitude function $v_{n}(\mathrm{x}, \mathrm{y}, \mathrm{t}) \quad\left(v_{-1}=0\right)$ :

(4. 4) $\quad \bar{\Phi}_{t}^{2}-\bar{\Phi}_{x}^{2}-\bar{\Phi}_{y}^{2}=0, \quad x \geqq 0, \quad 0 \leqq t \leqq T$,

(4. 5) $\left.\quad\left(\Phi_{y}+\varphi(y)^{2} \Phi_{x}\right)\right|_{x=0}=0, \quad 0 \leqq l \leqq T ;$

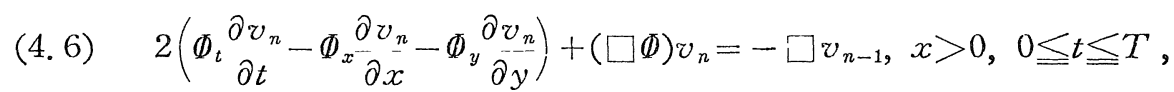

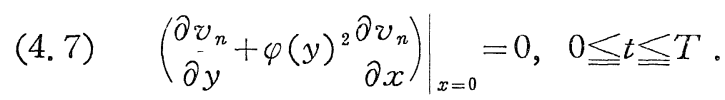

Combining (4.4) and (4.5) on the surface $\{x=0\}$, we have the equation for $l(y, t)=\left.\Phi\right|_{x=0}$ :

$$
l_{y}-\varphi^{2} \sqrt{ } l_{t}^{2}-l_{y}^{2}=0
$$

Here we do not use the other equation $l_{y}+\varphi^{2} \sqrt{ } l_{t}^{\overline{2}}-l_{y}^{2}=0$. As is easily seen, the function

$$
l(y, t)=\theta(y)+t
$$

is a solution of (4.8). By Hamilton-Jacobi's theory we have a solution $\Phi(x, y, t)$ of $(4.4)$ with $\left.\Phi\right|_{x=0}=l(y, t)$. Thus we obtain a real solution $\Phi(x, y, i)$ satisfying (4.4) and (4.5). (4.6) and (4.7) yield the following equation for $\widetilde{\mho}_{n}\left(\jmath^{\prime}, t\right)=\left.v_{n}\right|_{x=0}$ :

$$
\begin{gathered}
\left(\left.\Phi_{x}\right|_{x=0}-\left.\varphi^{2} \Phi_{y}\right|_{x=0}\right) \frac{\partial \widetilde{v}_{n}}{\partial y}+\left.\varphi^{2} \Phi_{t}\right|_{x=0} \frac{\partial \widetilde{v}_{n}}{\partial t}+\left.\frac{\varphi^{c} \square \Phi}{2}\right|_{x=0} \widetilde{v}_{n} \\
=-\left.\frac{\varphi^{2}}{2} \square v_{n-1}\right|_{x=0} .
\end{gathered}
$$

The characteristic curve of this equation thruugh $\left(0, t_{1}\right)$ coincides with 
the curve $\left\{(y, t): \theta(y)+t=t_{1}\right\}$ since $\left.\Phi_{x}\right|_{x=0}=-\sqrt{l_{t}^{2}-l_{y}^{2}},\left.\quad \Phi_{y}\right|_{x=0}=l_{y},\left.\Phi_{t}\right|_{x=0}$ $=l_{t}$ and $l(y, t)=\theta(y)+t$. Solving (4.6) for $x>0$ with $\left.v_{n}\right|_{x=0}=\widetilde{v}_{n}(y, t)$, we get $v_{n}(x, y, t)$ satisfying both (4.6) and (4.7). Note that every characteristic curve of (4.6) reaches in the direction $x>0, t>0$.

Now, let the mixed problem (1.1) have a finite propagation speed $v$. Then, if the data $\left(u_{0}, u_{1}, f, g\right)$ of (1.1) satisfy

$$
\left\{\begin{array}{l}
f(x, y, t)=0 \quad \text { on } \quad C_{t_{0}} \cap\left\{x>0,0<t<t_{0}\right\}, \\
g(y, t)=0 \quad \text { on } C_{t_{0}} \cap\left\{x=0,0<t<t_{0}\right\}, \\
u_{0}(x, y)=u_{1}(x, y)=0 \text { on } C_{t_{0}} \cap\{x>0, t=0\},
\end{array}\right.
$$

the solution $u(x, y, t)$ is equal to zero on $C_{t_{0}} \cap\left\{x>0,0<t<t_{0}\right\}$, where $C_{t_{0}}=\left\{(x, y, t): t-t_{0}+\frac{1}{v}\left(x^{2}+y^{2}\right)^{1 / 2}<0\right\}$. Since $\frac{d \theta}{d y}(0)=0$, we can choose a small constant $t_{0}(>0)$ such that $v t_{0} \leqq \frac{1}{3} \delta\left(\mathrm{t}_{0}\right) \quad($ where $\theta(\delta(t))=t)$. If $\left|t_{1}-t_{0}\right|$ is small enough, the intersection of $y$-axis and the characteristic curve of (4.10) through $\left(0, t_{1}\right)$ lies in $\left\{\frac{2}{3} \delta\left(t_{0}\right)<y<\frac{4}{3} \delta\left(t_{0}\right)\right\}$. Therefore, we can take $\left\{\widetilde{v}_{n}(y, t)\right\}_{n=0,1, \ldots}$ so that $\widetilde{v}_{n} \in C^{\infty}, \widetilde{v}_{0}\left(0, t_{0}\right) \neq 0$ and $\operatorname{supp}\left[\widetilde{v}_{n}(y, 0)\right]$ $\subset\left[\frac{2}{3} \delta\left(t_{0}\right), \frac{4}{3} \delta\left(t_{0}\right)\right]$. Hence, we can construct the asymptotic solution $u_{N}(x, y, t)$ satisfying

$$
\left\{\begin{array}{l}
\square u_{N}(x, y, t)=e^{i k \oplus} \square v_{N}(x, y, t)(i k)^{-N} \text { in } \mathbb{R}_{+}^{2} \times(0, T), \\
B u_{N}(y, t)=0 \quad \text { on } \quad \mathbb{R}^{1} \times(0, T), \\
\left.\frac{\partial u_{N}}{\partial t}\right|_{t=0}=\left.u_{N}\right|_{t=0}=0 \quad \text { on } \quad C_{t_{0}} \cap\{x>0, t=0\} .
\end{array}\right.
$$

Let $\chi(x)\left(\in C^{\infty}\right)=1$ for $0 \leqq x \leqq v T$ and $\chi(x)=0$ for $x \geqq v T+1$. Then, $f_{N}(x, y, t)=-e^{i k \omega} \square v_{N}(x, y, t) \chi(x)$ belongs to $H_{m+3}\left(\boldsymbol{R}_{+}^{2} \times(0, T)\right)$ (m $=6,7, \cdots)$. Furthermore, we have $B^{\partial^{j} f_{-}} \frac{\partial}{\partial t^{j}}=\left(\frac{\partial}{\partial y}+\varphi^{2} \frac{\partial}{\partial x}\right) \frac{\partial^{j}}{\partial t^{j}} f_{N \mid}^{l}=0$ for $j=0,1, \cdots$. Therefore the data $\left(u_{0}, u_{1}, f, g\right)=\left(0,0, f_{N}, 0\right)$ satisfy the compatibility condition of infinite order. By the result in $\S 3$, there exists a solution $w_{N}(x, y, t)\left(\in C^{\infty}\left(\overline{\mathbb{R}_{+}^{2}} \times[0, T]\right)\right)$ satisfying

$$
\left\{\begin{array}{l}
\square w_{N}(x, y, t)=f_{N}(x, y, t) \text { in } \mathbb{R}_{+}^{2} \times(0, T), \\
B w_{N}(y, t)=0 \text { on } \mathbb{R}^{1} \times(0, T), \\
\left.\frac{\partial w_{N}}{\partial t}\right|_{t=0}=\left.w_{N}\right|_{t=0}=0 \text { on } \mathbb{R}_{+}^{2},
\end{array}\right.
$$


and the estimate

$$
\sup _{(x, y, t) \in \mathbb{R}_{+}^{2} \times(0, T)}\left|w_{N}(x, y, t)\right| \leqq C_{1}\left\|f_{N}\right\|_{9, \mathbb{R}_{+}^{2} \times(0, T)} \leqq C_{2} k^{9}
$$

holds, where the constant $C_{2}$ does not depend on $k(>0)$. We take the integer $N>9$, and set

$$
u(x, y, t)=w_{N}(x, y, t)(i k)^{-N}+u_{N}(x, y, t) .
$$

Then, $\left(u_{0}, u_{1}, f, g\right)=\left(\left.u\right|_{t=0},\left.\frac{\partial u}{\partial t}\right|_{t=0}, \square u, B u\right)$ satisfies (4.11), but $u(x, y, t)$ does not equal zero in a neighborhood of $(x, y, t)=\left(0,0, t_{0}\right)$ when $k>0$ is large enough. This is a contradiction. Therefore, (i) of the theorem is proved.

Remark 4.2. (i) of Theorem 4.1 can be verified also by the methods Ikawa [3] has employed to study the propagation speed of the mixed problem.

\section{§5. A Non-well-posed Case}

In this section we shall prove the following

Theonem 5. 1. Let the function $\psi(y)$ in (1.1) satisfy $\psi(0)=0$, $\psi(y)>0$ for $y<0$ and $\psi(y)<0$ for $y>0$. Then the problem (1.1) is not well-posed.

Here 'well-posed' means that there exists a unique solution of (1.1) in $\left.C^{\infty} \overline{\left(\mathbb{R}_{+}^{2}\right.} \times[0, T]\right)$ for any $\left(u_{0}, u_{1}, f, g\right) \in C^{\infty}\left(\overline{\mathbb{R}_{+}^{2}}\right) \times C^{\infty}\left(\overline{\mathbb{R}_{+}^{2}}\right) \times C^{\infty}\left(\overline{\mathbb{R}_{+}^{2}}\right.$ $\times[0, T]) \times C^{\infty}\left(\mathbb{R}^{1} \times[0, T]\right)$ satisfying the compatibility condition of infinite order.

Proof. If (1.1) is well-posed, there exist a positive integer $l$ and a compact set $K\left(\subset \overline{\mathbb{R}_{+}^{2}}\right)$ such that

$$
\begin{aligned}
\sup _{0 \leqq t \leqq T}|u(0,0, t)| \leqq & C\left(|\square u|_{l, K \times[0, T]}+|B u|_{l, K^{\prime} \times[0, T]}\right. \\
& \left.+\left.u\right|_{t=0}+\left|\frac{\partial u}{\partial t}\right|_{t=0}||_{l, K}\right)
\end{aligned}
$$


where $K^{\prime}=K \cap\{x=0\}$ and the semi norm $|v|_{l, M}$ denotes $\sum_{|\alpha| \leqq l} \sup _{x^{\prime} \in M I}\left|\left(\frac{\partial}{\partial x^{\prime}}\right)^{\alpha} v\left(x^{\prime}\right)\right|$. Let us construct an aymptotic solution

$$
\iota_{N}(x, y, t)=\sum_{n=0}^{N} e^{i k \Phi(x, y, t)} v_{n}(x, y, t)(i k)^{-n}
$$

which is of the same type as in the proof of Theorem 4.1 and breaks the inequality (5.1) as $k \rightarrow+\infty$. By the same procedure as in the proof of Theorem 4.1, we get the following equations (cf. (4. 4) (4.7)):

$$
\begin{aligned}
& \Phi_{t}^{2}-\Phi_{x}^{2}-\Phi_{y}^{2}=0, \quad x>0, \quad 0 \leqq t \leqq T, \\
& \left.\left(\Phi_{y}+\psi(y) \Phi_{x}\right)\right|_{x=0}=0, \quad 0 \leqq t \leqq T ; \\
& 2\left(\Phi_{t} \frac{\partial v_{n}}{\partial t}-\Phi_{x} \frac{\partial v_{n}}{\partial x}-\Phi_{y}{ }^{\partial v_{n}} \frac{\partial y}{\partial y}\right)+(\square \Phi) v_{n}=-\square v_{n-1} \quad\left(v_{-1}=0\right), \\
& x>0,0 \leqq t \leqq T, \\
& \left.\left(\frac{\partial r_{n}^{\prime}}{\partial y}+\psi(y) \frac{\partial v_{n}}{\partial x}\right)\right|_{x=0}=0,0 \leqq t \leqq T
\end{aligned}
$$

We have a real solution $\Phi(x, y, t)$ of (5.2) and (5.3) with $\Phi_{x}<0, \Phi_{t}>0$. Combining (5.4) and (5.5), we obtain the equation for $\widetilde{v}_{n}(y, t)=\left.v_{n}\right|_{x=0}$ :

$$
\begin{gathered}
\left(\left.\Phi_{x}\right|_{x=0}-\left.\psi \Phi_{y}\right|_{x=0}\right) \frac{\partial \widetilde{v}_{n}}{\partial y}+\left.\psi \Phi_{t}\right|_{x=0} \frac{\partial \widetilde{v}_{n}}{\partial t}+\frac{\left.\phi \square \Phi\right|_{x=0}}{2} \widetilde{v}_{n} \\
=-\left.\frac{\psi}{2}\left(\square v_{n-1}\right)\right|_{x=0} .
\end{gathered}
$$

From the assumption of Theorem 5.1, any characteristic curve $t=\tilde{t}(y)$ of (5.6) is concave (i.e. $\frac{d \tilde{t}}{d y}(y)<0$ for $-\delta_{1}<y<0$ and $\frac{d \tilde{t}}{d y}(y)>0$ for $0<y<\delta_{1}$, where $\delta_{1}$ is a small positive constant). Therefore we can choose the $\left\{v_{n}(x, y, t)\right\}_{n=0,1,2, \ldots}$ so that $v_{n}(x, y, t) \in C^{\infty}\left(\overline{\mathbb{R}}_{+}^{2} \times[0, T]\right) \quad(n=0,1,2$, $\cdots), v_{0}\left(0,0, t_{0}\right) \neq 0$ for some $i_{0}\left(0<t_{0}<T\right)$ and $v_{n}(x, y, 0)=\frac{\partial v_{n}}{\partial t}(x, y, 0)$ $=0$ on $\mathbb{R}_{+}^{2}(n=0,1, \cdots)$. Then, $u_{N}(x, y, t)=\sum_{n=0}^{N} e^{i k \emptyset} v_{n}(i k)^{-n}$ satisfies

$$
\left\{\begin{array}{l}
\square u_{N}(x, y, t)=e^{i k \cdot} \square v_{N}(i k)^{-N} \text { in } \mathbb{R}_{+}^{2} \times(0, T), \\
B u_{N}(y, t)=0 \text { on } \mathbb{R}^{1} \times(0, T), \\
\left.\frac{\partial u_{N}}{\partial t}\right|_{t=0}=\left.u_{N}\right|_{t=0}=0 \text { on } \mathbb{R}_{+}^{2},
\end{array}\right.
$$


and $\left|u_{N}\left(0,0, t_{0}\right)\right| \rightarrow\left|v_{0}\left(0,0, t_{0}\right)\right| \neq 0$ as $k \rightarrow+\infty$. This violates (5. 1) (if $N>l)$. The proof is complete.

\section{References}

[1] Caiderón, A. P. and Vaillancourt, R., A class of bounded pseudo-differential operators, Proc. Nat. Acad. Sci. USA, 69 (1972), 1185-1187.

[2] Ikawa, M., Mixed problem for the wave equation with an oblique derivative boundary condition, Osaka J. Mati. 7 (1970), 495-525.

[3] Ikawa, M., Problèmes mixtes pour l'équation des ondes II Publ. RIMS, Kyoto Univ. 13 (1977), 61-106.

[4] Kajitani, K., A necessary condition for the well posed hyperbolic mixed problem with variable coefficients, J. Math. Kyoto Univ. 14 (1974), 231-242.

[5] Keller, J. B., Lewis, R. M. and Seckler, B. D., Asymptotic solution of some diffraction problems, Comm. Pure Appl. Math. 9 (1956), 207-265.

[6] Kumano-go, H., Pseudo-differential operators (in Japanese), Iwanami, Tokyo, 1975.

[7] Sakamoto, R., $\mathcal{E}$-well posedness for hyperbolic mixed problems with constant coefficients, J. Math. Kyoto Univ. 14 (1974), 93-118.

[8] Tsuji, M., Characterization of the well-posed mixed problem for wave equation in a quarter space, Proc. Japan Acad., 50 (1974), 138-142. 
